\title{
On the Emergence of Social Information Science in China and Our Research Outline ${ }^{\dagger}$
}

\author{
Kang Ouyang \\ Institute of Philosophy and the Institute for Social Information Science, Huazhong University of Science and \\ Technology, Wuhan 430074, China; kouyang@mail.hust.edu.cn; Tel.: +86-27-8755-3020; Fax: +86-27-8754-5438 \\ + Presented at the IS4SI 2017 Summit DIGITALISATION FOR A SUSTAINABLE SOCIETY, Gothenburg, \\ Sweden, 12-16 June 2017.
}

Published: 9 June 2017

Social Information Science (or Social Informatics) is a new and inter-discipline branch subject in China and even in the world. This paper probe the emergence of social information science and the research outline of us.

I. The proposal of the social information science. We set up the research from an extension from the theoretical informatics to the concrete informatics; an internal bond of integrating various subjects in human and social sciences; an intersection and mutual permeation between the social science and the natural science; an intersection and interaction among human and social sciences, a modern information science and information technology; a strengthening to the research into the Social Epistemology.

II. On the concept of social information. Social information directly is different with self-existent and natural information, and more related to human's autonomous creative activities, to society's culture inheritance, to social value, to human's spiritual interaction and to human's emotions.

III. On the theoretical orientation of the social information science. Social Information Science is a concrete branch of informatics, a generation of sub-disciplines of social information, a kind of traversing and comprehensive research on individual social science from the angle of information, a kind of exchange and interaction between social theoretical research and the modern information technology.

IV. The research focus of the social information science. The paper lists 10 main focuses in the research of social information science. Such as the interweaving of the factual information and the valuable information, of the subjective information and the objective information, of the rational information and irrational information, of the general information and the idiosyncratic information; the difference between comprehensive information and restrictive information, the collision between purpose-oriented information and spontaneous information, the man-made increase and decrease and purposeful distortion of social information, the cognition, evaluation, interaction, intersection and even mutual restriction of information, the differences between the exposition and understanding of information, the different effects resulting from the passive reception of information and the active collection of information

$\mathrm{V}$. The system and frame of the social information science. In general, there should be four levels of researches if the social information science is to be viewed as a relatively independent subject: the philosophical level, the scientific theoretical level, concrete apply level, social information technology and methods.

(C) 2017 by the author. Licensee MDPI, Basel, Switzerland. This article is an open access article distributed under the terms and conditions of the Creative Commons Attribution (CC BY) license (http://creativecommons.org/licenses/by/4.0/). 